\title{
THEORETICAL STUDY OF THE FLOW AND TEMPERATURE FIELDS IN CZ SINGLE CRYSTAL GROWTH
}

\author{
TAKAO TSUKADA, NOBUYUKI IMAISHI AND MITSUNORI HOZAWA \\ Chemical Research Institute of Non-Aqueous Solutions, Tohoku University, Sendai 980
}

\begin{abstract}
Key Words: Crystal Growth, Silicon, Sapphire, Czochralski Method, Flow Pattern, Temperature Distribution, Interface Shape, Numerical Simulation, Finite Element Method

For the $\mathrm{CZ}$ crystal growth of silicon and sapphire, the flow and temperature fields with non-flat melt/crystal and melt/gas interfaces were studied theoretically by use of the finite element method.

The theoretical method used here can predict the temperature distribution and the flow pattern (forced convection, free convection, Marangoni convection and their combined flow) in the melt and crystal, including the shapes of the melt/crystal and melt/gas interfaces and the crystal radius, although calculation was limited to the case of small $\mathrm{CZ}$ apparatus.

It is found that the melt/gas interface shape affects the flow pattern in the melt, and that the melt/crystal interface shape for a system of small $\mathrm{Pr}$ such as silicon is not sensitive to the flow field. But for a system of larger $\mathrm{Pr}$ such as sapphire, the melt/crystal interface shape is strongly dependent on the flow field.

It is also found that the Marangoni effect, if it operates, plays the most important roll in promoting the flow in the melt.
\end{abstract}

\section{Introduction}

The Czochralski (CZ) method is applied to the growth of single crystals utilized in semiconductor devices or acousto-optic devices. For the production of perfect single crystals, it is necessary to control or suppress thermal convection in the melt. In the industrial $\mathrm{CZ}$ apparatus, the crystal and/or the crucible are generally rotated in order to improve thermal symmetry around the crystal and also to homogenize the dopant distribution. Accordingly, the various flows, free convection, thermocapillary flow and forced convection caused by crystal and crucible rotation coexist in the melt, and thus it is very important to acquire correct knowledge about the flow pattern and the mechanism of heat transfer in the melt and the crystal in order to control the quality of crystals. In the crystal growth of oxide, it is wellknown that the melt/crystal interface changes shape abruptly from concave to convex toward the crystal with increasing crystal rotation rate. This means that the temperature field in a melt of oxide with large Prandtl number is strongly influenced by the flow pattern in the melt, and thus it is necessary to know the responses of melt/crystal interface shape and crystal radius against the various processing parameters such as crystal rotation rate and crucible temperature.

One of the methods used to predict the flow pattern in the melt is computer simulation. Crochet et al., ${ }^{3)}$

\footnotetext{
Received July 4, 1987. Correspondence concerning this article should be addressed to
} T. Tsukada.
Kobayashi, ${ }^{7-12)}$ Langlois $^{13-16)}$ and Mihelcic et $a l .{ }^{17-19)}$ computed the temperature distribution and the flow pattern in the melt.

In the previous works, however, emphasis has been placed on finding out the qualitative aspects of melt flow in very simplified circumstances. These simulations cannot provide the practically important quantities such as the crystal radius, crystal/melt interface shape, distributions of temperature and velocities in the melt and crystal under a given processing condition. There is an urgent need among crystalpull engineers for the development of a practical numerical simulation shceme in which all components of the $\mathrm{CZ}$ furnace, i.e. the crystal, the melt pool, the crucible and the ambient walls, are taken into the analysis so as to predict those quantities. Recently, Derby et al., ${ }^{4}$ Srivastava et al. ${ }^{21)}$ and Tsukada et $a .^{22,23)}$ have successfully applied finite-element analysis to the conduction-dominated problem for $\mathrm{CZ}$ growth, and have simulated numerically the interface shapes, crystal radius, and temperature distribution. In these analyses, however, melt convection was not taken into account.

The aim of this work is to develop such a practical simulation scheme in which the flow in the melt is also taken into the analysis. The scheme is used to reveal the effects of several factors on the $\mathrm{CZ}$ pulling of silicon and $\mathrm{Al}_{2} \mathrm{O}_{3}$ (sapphire).

\section{Theory}

Figure 1 shows the cylindrical coordinates used here. 


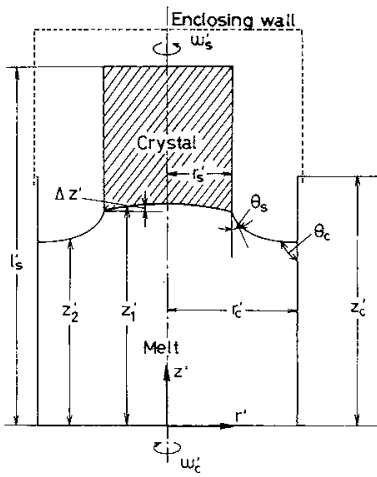

Fig. 1. Coordinate system

The following assumptions are made in the numerical simulations. 1) The system is in a pseudo-steady state and is rotationally symmetric. 2) The fluid is incompressible. 3) The physical properties are constant and are evaluated at the melting-point temperature, except for the density and surface tension, which are dependent on the temperature to allow for the effect of buoyancy and thermocapillary forces. 4) Heat transfer in the crystal is dominated by conduction, while in the melt it is dominated by conduction and convection. 5) The crystal is pulled continuously from the melt at a constant rate. 6) The crucible and the ambient wall are maintained at respective constant temperatures. 7) Heat loss from the surfaces of melt and crystal is due to radiation alone. 8) The melt/crystal interface is at the meltingpoint temperature. 9) The contact angle of melt against the crystal and the crucible is constant.

Under the above assumptions, the dimensionless forms of the Navier-Stokes equation with the Boussinesq approximation and the continuity equation for the melt are given as follows, where the characteristic length is crucible radius $r_{c}^{\prime}$.

$$
\begin{gathered}
\boldsymbol{v}_{l} \cdot \boldsymbol{\nabla} \boldsymbol{v}_{l}=-\nabla p_{l}-\nabla \cdot \tau_{l}+\operatorname{Gr}\left(T_{l}-1\right) \boldsymbol{e}_{z} \\
\boldsymbol{\nabla} \cdot \boldsymbol{v}_{l}=0
\end{gathered}
$$

where $\tau$ is the stress tensor, defined as $-\left(\nabla \boldsymbol{v}_{l}+\nabla \boldsymbol{v}_{l}^{T}\right)$.

The dimensionless energy equations for the melt and the crystal are described by Eqs. (3) and (4).

$$
\begin{aligned}
& P r v_{l} \cdot \nabla T_{l}=\nabla^{2} T_{l} \\
& P e e_{z} \cdot \nabla T_{s}=\nabla^{2} T_{s}
\end{aligned}
$$

where suffixes $l$ and $s$ mean the melt and the crystal respectively, and $P e$ corresponds to the dimensionless pull rate.

The boundary conditions are expressed as follows.

At both the side wall and the bottom of the crucible:

$$
u_{l}=v_{l}=0, \quad w_{l}=r \operatorname{Re}_{c}, \quad T_{l}=T_{\mathrm{c}}
$$

At the melt/gas interface:

$$
\begin{aligned}
& \boldsymbol{n} \cdot \boldsymbol{v}_{l}=0, \quad \boldsymbol{\tau}_{l}: \boldsymbol{n} \boldsymbol{t}=\operatorname{Ma} \boldsymbol{\nabla} T_{l} \cdot \boldsymbol{t} \\
& \boldsymbol{\tau}_{l}: \boldsymbol{n} \boldsymbol{e}_{\theta}=0, \quad-\boldsymbol{n} \cdot \boldsymbol{\nabla} T_{l}=q_{\mathrm{rad}, l}
\end{aligned}
$$

At the melt/crystal interface:

$$
\begin{aligned}
& u_{l}=v_{l}=0, \quad w_{l}=r \operatorname{Re}_{s} \\
& \boldsymbol{n} \cdot \nabla T_{l}-\kappa \boldsymbol{n} \cdot \nabla T_{s}=\kappa \text { PeSt } \boldsymbol{n} \cdot \boldsymbol{e}_{z} \\
& T_{l}=T_{s}=1
\end{aligned}
$$

At the crystal side and top:

$$
-\boldsymbol{n} \cdot \boldsymbol{\nabla} T_{s}=q_{\mathrm{rad}, \boldsymbol{s}}
$$

At the center line:

$$
\begin{aligned}
& u_{l}=w_{l}=0 \\
& \boldsymbol{n} \cdot \nabla \boldsymbol{v}_{l}=\boldsymbol{n} \cdot \nabla T_{l}=\boldsymbol{n} \cdot \nabla T_{s}=0
\end{aligned}
$$

where $u_{l}, v_{l}$ and $w_{l}$ are the radial, axial and azimuthal components of velocity vector $\boldsymbol{v}_{l}$, respectively. $q_{\mathrm{rad}, k}$ $(k=l$ and $s)$ is heat loss per unit area on the boundary surfaces due to radiation, taking into account both directed and reflected radiation heat exchange between an individual surface element and all surrounding surfaces, and is given as follows. ${ }^{21,221}$

$$
q_{\mathrm{rad}, k}=R_{k}\left(T_{k}^{4}-\left(\sum_{j=1}^{N} A_{j} \varepsilon_{j} T_{j}^{4} G_{j k}\right) / A_{k} / \varepsilon_{k}\right)
$$

where $R_{\mathrm{k}}$ is radiation number, $N$ is total number of radiation surface elements present and $G_{j k}$ is Gebhart's absorption factor, ${ }^{21)}$ which is the fraction of emission from surface $A_{j}$ that reaches $A_{k}$ and is absorbed.

Accordingly, the flow and temperature fields are given by solving Eqs. (1)-(4) under the boundary conditions.

The melt/crystal interface shape $z_{1}$ is determined such that Eq. (5-13) is satisfied, i.e. the interface is at the melting-point isotherm.

The melt/gas interface shape $z_{2}$ and the crystal radius are obtained by solving the Young-Laplace equation, Eq. (7), under the following conditions.

$$
2 \mathrm{H}=\mathrm{Boz}_{2}+\lambda
$$

The boundary conditions are expressed as

$$
\begin{array}{lll}
\theta=\theta_{s} & \text { at } & r=r_{s} \\
\theta=\theta_{c} & \text { at } & r=1
\end{array}
$$

The melt volume is given by Eq. (9).

$$
V_{m}=\int_{0}^{r_{s}} z_{1} r d r+\int_{r_{s}}^{1} z_{2} r d r
$$

To solve the above problem, the Galerkin finiteelement method ${ }^{3,4,21-23)}$ is used. The melt and crystal are discretized by isoparametric elements consisting of a nine-noded quadrilateral as shown in Fig. 2, 


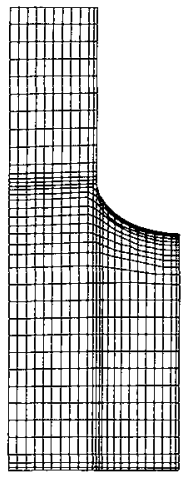

Fig. 2. Discretization of calculation domain for the finite element method

where the total number of elements and nodal points are 719 and 2999, respectively.

In each element, velocity vector $v$ and temperature $T$ are approximated with the biquadratic trial function $\phi_{i}$ as follows.

$$
\begin{aligned}
\boldsymbol{v}(r, z) & =\sum \phi_{i} \boldsymbol{v}_{i} \\
T(r, z) & =\sum \phi_{i} T_{i}
\end{aligned}
$$

The bilinear trial function $\varphi_{k}$ is used to approximate pressure $p$.

$$
p(r, z)=\sum \varphi_{k} p_{k}
$$

The shape of the gas/melt interface is expressed by the following equation.

$$
z_{2}(r)=\sum \chi_{j} z_{j}
$$

where $\chi_{j}$ is the Hermite cubic trial function.

By substituting Eqs. (10)-(13) into Eqs. (1)-(4) and (7), the Galerkin procedure gives the set of algebraic equations. The equations are solved by use of the Newton-Raphson scheme. The calculations for the fields, crystal radius, and interface shapes are repeated until the cut-off error is less than a given value.

\section{Results and Discussion}

We computed the flow and temperature fields in the melt and the crystal, and the shapes of melt/crystal and melt/gas interfaces for the two systems: silicon as an example of systems with small $\operatorname{Pr}(\operatorname{Pr}=0.014)$ and $\mathrm{Al}_{2} \mathrm{O}_{3}$ (sapphire) as an example of those with relatively large $\operatorname{Pr}(\operatorname{Pr}=0.34)$. The calculations were carried out for cases with the melt pool in a small cruicible $(20 \mathrm{~mm}$ in diameter) for both silicon and $\mathrm{Al}_{2} \mathrm{O}_{3}$. Physical properties and processing parameters used in numerical simulations are listed in Table 1. In the following Sections 2.1-2.3, we describe the effects of crystal rotation, exposure to hot cruicible wall, and non-flat interfaces, respectively. In these sections, the thermocapillary effect is ignored by setting $M a=0$ in Eq. (5-6), for the sake of comparison with the previous works. The effect of thermocapillary force is

\begin{tabular}{|c|c|c|}
\hline & $\mathrm{Si}$ & $\mathrm{Al}_{2} \mathrm{O}_{3}$ \\
\hline Melting point $\left(T_{m}^{\prime}\right)[\mathrm{K}]$ & 1683 & 2316 \\
\hline Heat of solidification $\left(\Delta H_{s}\right)\left[\mathrm{J} \cdot \mathrm{g}^{-1}\right]$ & 1800 & 1046 \\
\hline \multicolumn{3}{|l|}{ Thermal conductivity } \\
\hline melt $\left(k_{l}\right)\left[\mathrm{W} \cdot \mathrm{cm}^{-1} \mathrm{~K}^{-1}\right]$ & 0.64 & 0.1 \\
\hline crystal $\left(k_{s}\right)\left[\mathrm{W} \cdot \mathrm{cm}^{-1} \mathrm{~K}^{-1}\right]$ & 0.22 & 0.1 \\
\hline \multicolumn{3}{|l|}{ Density } \\
\hline melt $\left(\rho_{l}\right)\left[\mathrm{g} \cdot \mathrm{cm}^{-3}\right]$ & 2.42 & 3.05 \\
\hline crystal $\left(\rho_{s}\right)\left[\mathrm{g} \cdot \mathrm{cm}^{-3}\right]$ & 2.3 & 4.0 \\
\hline Kinematic viscosity $\left(v_{l}\right)\left[\mathrm{cm}^{2} \cdot \mathrm{s}^{-1}\right]$ & 0.0036 & 0.00892 \\
\hline \multicolumn{3}{|l|}{ Heat capacity } \\
\hline melt $\left(C_{p l}\right)\left[\mathrm{J} \cdot \mathrm{g}^{-1} \mathrm{~K}^{-1}\right]$ & 1.0 & 1.26 \\
\hline crystal $\left(C_{p s}\right)\left[\mathrm{J} \cdot \mathrm{g}^{-1} \mathrm{~K}^{-1}\right]$ & 1.0 & 1.26 \\
\hline \multicolumn{3}{|l|}{ Emissivity } \\
\hline melt $\left(\varepsilon_{l}\right)$ & 0.3 & 0.33 \\
\hline crystal $\left(\varepsilon_{s}\right)$ & 0.46 & 0.33 \\
\hline crucible wall $\left(\varepsilon_{c}\right)$ & 0.59 & 0.3 \\
\hline ambient wall $\left(\varepsilon_{a}\right)$ & 0.8 & 0.3 \\
\hline \multicolumn{3}{|l|}{ Thermal expansion coefficient } \\
\hline$(\beta)\left[\mathrm{K}^{-1}\right]$ & $1.41 \times 10^{-5}$ & $3.0 \times 10^{-5}$ \\
\hline Surface tension $(\gamma)\left[\mathrm{dyn} \cdot \mathrm{cm}^{-1}\right]$ & 720 & 700 \\
\hline \multicolumn{3}{|l|}{$\begin{array}{l}\text { Temperature coefficient of surface } \\
\text { tension }\end{array}$} \\
\hline$\left(-d \gamma / d T^{\prime}\right)\left[\mathrm{dyn} \cdot \mathrm{cm}^{-1} \mathrm{~K}^{-1}\right]$ & 0.43 & 0.06 \\
\hline \multicolumn{3}{|l|}{ Contact angle } \\
\hline against crystal $\left(\theta_{s}\right)[\mathrm{deg}]$ & 11 & 17 \\
\hline against crucible $\left(\theta_{c}\right)[\mathrm{deg}]$ & 90 & 90 \\
\hline Prandtl number $[-]$ & 0.014 & 0.34 \\
\hline \multicolumn{3}{|l|}{ 2. Processing parameter } \\
\hline Crucible radius $\left(r_{c}^{\prime}\right)[\mathrm{cm}]$ & 1.0 & 1.0 \\
\hline Crucible height $\left(z_{c}^{\prime}\right)[\mathrm{cm}]$ & 1.9 & 1.9 \\
\hline Crystal length $\left(l_{s}^{\prime}\right)[\mathrm{cm}]$ & 2.7 & 2.7 \\
\hline Melt volume $\left(V_{m}^{\prime}\right)\left[\mathrm{cm}^{3}\right]$ & 4.7 & 4.7 \\
\hline Enclosing wall area $\left(A_{a}^{\prime}\right)\left[\mathrm{cm}^{2}\right]$ & 10.0 & 10.0 \\
\hline Enclosing wall temperature & 673 & 1737 \\
\hline \multirow[t]{2}{*}{ Crucible temperature $\left(T_{c}^{\prime}\right)[\mathrm{K}]$} & 1718 & 2457 \\
\hline & or 1737 & -2473 \\
\hline \multicolumn{3}{|l|}{ Crystal rotation rate } \\
\hline \multicolumn{3}{|l|}{ Crucible rotation rate } \\
\hline$\left(n_{c}^{\prime}\right)\left[\min ^{-1}\right]$ & 0 & 0 \\
\hline Pull rate $\left(V_{s}^{\prime}\right)\left[\mathrm{mm} \cdot \min ^{-1}\right]$ & 1.0 & 0.1 \\
\hline
\end{tabular}

Table 1. Physical properties and processing parameters used for calculations

1. Physical properties

discussed in 2.4.

\subsection{Effect of crystal rotation}

Figure 3 shows the velocity vectors and temperature distributions in the melt and the crystal when the crystal is rotated alone $\left(R e_{c}=0\right)$, where (a) is for those in silicon $\left(G r=1.8 \times 10^{6}\right)$ and (b) is for those in $\mathrm{Al}_{2} \mathrm{O}_{3}$ $\left(G r=8.56 \times 10^{5}\right)$. For $R e_{s}=0$, only the counterclockwise vortex caused by free convection is present in the melt. When the crystal is set in rotation, the flow in the melt splits into two vortices, one caused by free convection and the other by forced convection due to crystal rotation. When the rotation rate of crystal rod is slow, such as for $R e_{s}=300$ in silicon and $R e_{s}=350$ 


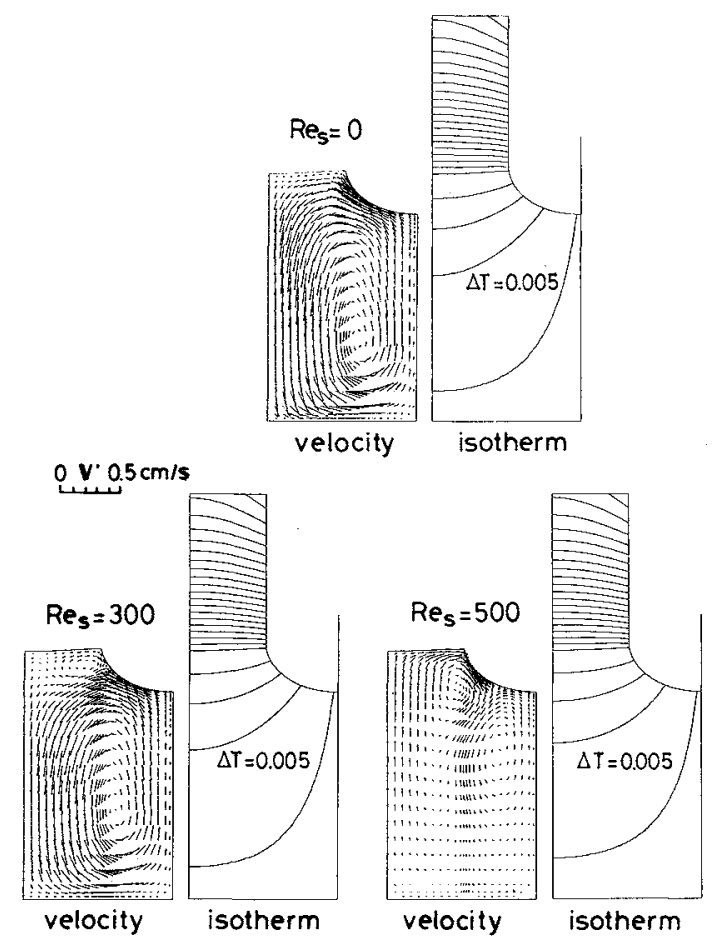

(a): Silicon crystal growth with $G r=1.8 \times 10^{6}, T_{c}=1.0207$, $R e_{c}=0$ and $M a=0$
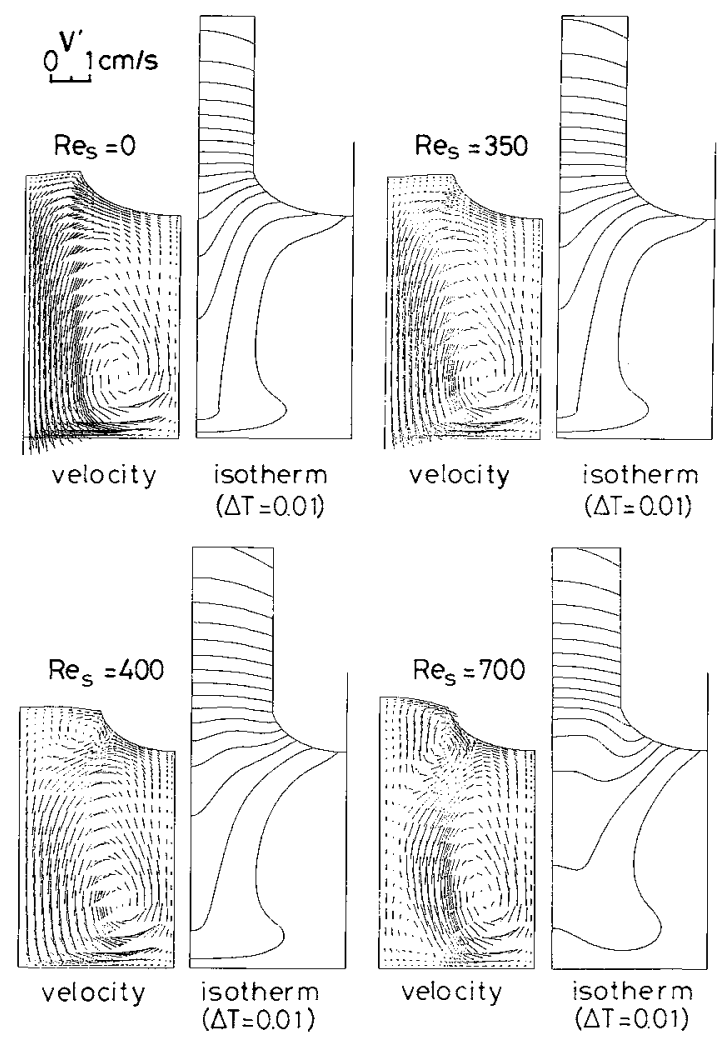

(b): Sapphire crystal growth with $G r=8.56 \times 10^{5}, T_{c}=1.068$, $R e_{c}=0$ and $M a=0$

Fig. 3. Effect of crystal rotation on flow pattern and temperature distribution in the melt and the crystal.

in $\mathrm{Al}_{2} \mathrm{O}_{3}$, the clockwise vortex due to crystal rotation exists only under the crystal rod, and the counterclockwise vortex prevails almost throughout the melt

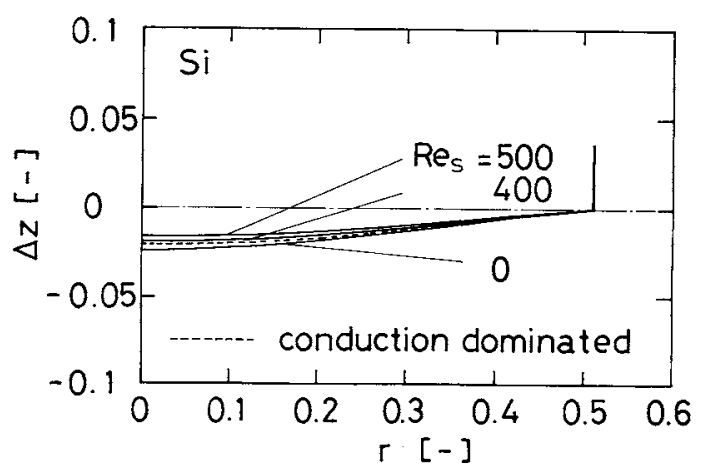

(a): Silicon crystal growth with $G r=1.8 \times 10^{6}, T_{c}=1.0207$, $R e_{c}=0$ and $M a=0$

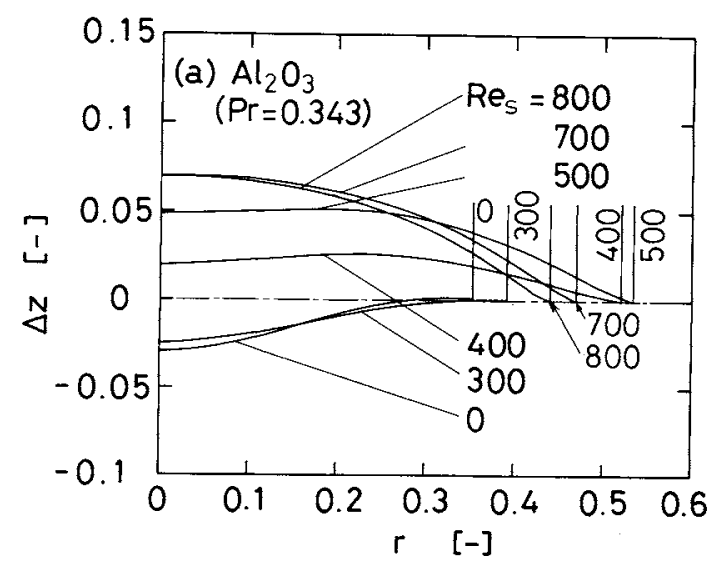

(b): Sapphire crystal growth with $G r=8.56 \times 10^{5}, T_{c}=1.068$, $R e_{c}=0$ and $M a=0$

Fig. 4. Effect of crystal rotation on melt/crystal interface shape.

pool-i.e. free convection is dominant. As $R e_{s}$ becomes larger $\left(R e_{s}=500\right.$ in silicon and $R e_{s}=700$ in $\mathrm{Al}_{2} \mathrm{O}_{3}$ ), however, the clockwise flow due to forced convection reaches down close to the crucible bottom. It is seen from these figures that flow patterns in the melt change with increasing $R e_{s}$ in almost the same way for both silicon and $\mathrm{Al}_{2} \mathrm{O}_{3}$. On the other hand, the effects of the flow pattern on the temperature distributions in the melt are different between (a) and (b). The Prandtl number of silicon melt is very small and thus the temperature field is only slightly affected by the flow field. In $\mathrm{Al}_{2} \mathrm{O}_{3}$, however, the temperature field is strongly influenced by the flow pattern, and thus the melt/crystal interface shape and crystal radius also change with $R e_{s}$.

Figure 4 shows the effect of the crystal rotation on the shape of the melt/crystal interface and the crystal radius for (a) silicon and (b) $\mathrm{Al}_{2} \mathrm{O}_{3}$ ctystal growth, where $\Delta z$ represents the difference between $z_{1}$ and $z_{1}$ at $r=r_{s}$ as shown in Fig. 1. For silicon as shown in (a), the melt/crystal interface shape and the crystal radius is practically insensitive to $R e_{s}$, and is similar to that calculated by the conduction-dominated model. This is due to the small $\mathrm{Pr}$ of silicon melt. For $\mathrm{Al}_{2} \mathrm{O}_{3}$, as 
shown in Fig. 4(b), the interface shape and the crystal radius is strongly influenced by $R e_{s}$, i.e. the flow pattern in the melt. In this case, the conductiondominated model gives $r_{s}=0.645$ and $\Delta z$ (at $r=0$ ) $=$ -0.0288 . When free convection is dominant, the melt crystal interface is convex to the melt, but it reverses to become convex to the crystal at $R e_{s} \doteqdot 400$. This abrupt change of melt/crystal interface shape has been observed experimentally during the crystal growth processes of oxides, ${ }^{2)}$ and has been believed to be due to change of dominant flow in the melt from free convection to forced convection.

\subsection{Effect of hot crucible wall}

So far, both directed and reffected radiation heat exchange between an individual surface element and all surrounding surfaces have been taken into account to evaluate the heat loss due to radiation on the boundary surfaces. In most previous works, however, heat loss was evaluated using only the effective temperature $T_{e}$ of the surroundings as follows.

$$
q_{\mathrm{rad}, k}=R_{k}\left(T_{k}^{4}-T_{e}^{4}\right)
$$

where the existence of the exposed hot crucible wall was not taken into account.

Figure 5 shows the flow patterns and temperature distributions around a crystal rod of given radius. In case (a), Eq. (6) is used for the radiative boundary condition, while in case (b), Eq. (14) is used. In Fig. 5, $G r=1.8 \times 10^{6}$ and $R e_{s}=500$ for silicon crystal growth are used. When the exposed hot crucible wall is ignored, radiative heat loss from the crystal rod and the melt surface is larger than that when the hot crucible wall is considered. Accordingly, to keep the crystal radius constant a higher crucible temperature is required. The intensity of counterclockwise vortex due to free convection in (b) becomes stronger than that in (a), and the area occupied by the clockwise flow due to crystal rotation is smaller. This is simply due to the larger temperature difference between the crucible and the crystal rod in case (b).

\subsection{Effect of non-flat interfaces}

Figure 6 shows the flow pattern and temperature distribution in the melt when both the melt/gas and melt/crystal interfaces are assumed to be flat. This calculation was made to reveal the effect of interface shape on the flow field. The dimensionless parameters in Fig. 6 are similar to those in Fig. 5(b) and heat loss due to radiation is evaluated by using Eq. (14). Under the assumption of flat interfaces, the clockwise flow near the melt/crystal interface caused by crystal rotation is weaker and free convection is more dominant than in the results shown in Fig. 5(b). The reason for this difference is that in the case of a curved melt/gas interface the counterclockwise flow caused by free convection cannot ascend into the pulled-up meniscus region, but separates from the gas/melt interface and

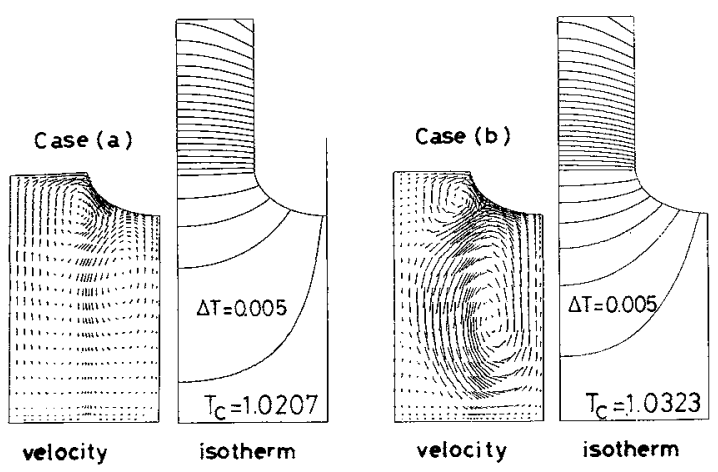

Fig. 5. Effect of the exposed hot crucible wall on the flow pattern in silicon melt around a crystal rod of the same diameter. With $G r=1.8 \times 10^{6}, R e_{c}=0$ and $M a=0$

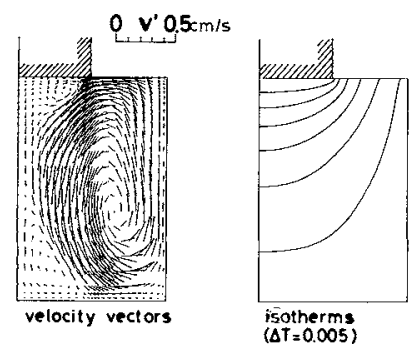

Fig. 6. Flow pattern and temperature distribution when the melt/crystal and melt/gas interfaces are flat. With $R e_{s}=500$, $R e_{c}=M a=0, G r=1.8 \times 10^{6}$ and $T_{c}=1.0323$

returns to the bulk of the melt at a point lower than and away from the crystal edge. Forced convection prevails in the meniscus region. In the case of flat interfaces, by contrast, the free convection reaches very close to the crystal edge and the forced convection is confined to a small region under the crystal. The effect of non-flat melt/gas interface is important, especially in a small crucible as in the present cases.

These sample calculations, shown in $\mathbf{2 . 2}$ and $\mathbf{2 . 3}$, reveal that the simulation of melt convection has significance only when all the influential factors are correctly taken into account.

\subsection{Effect of thermocapillary force}

In addition to forced and free (buoyancy-driven) convection, the thermocapillary flow (Marangoni convection) should be also taken into account. However, the reported values of $\left(\partial \gamma / \partial T^{\prime}\right)^{14,15)}$ of hightemperature melt do not seem entirely reliable. ${ }^{15)}$ In this report, the effect of the thermocapillary force is studied by setting $M a$, in other words $\left(\partial \gamma / \partial T^{\prime}\right)$, as an arbitrary parameter. Figure 7 shows the effect of Marangoni convection on the flow field in the melt for (a) silicon and (b) $\mathrm{Al}_{2} \mathrm{O}_{3}$ crystal growth. The figures show the change of flow pattern with increasing Marangoni number $M a$. Here the crystal alone is rotated $\left(R e_{c}=0\right)$. The intense flow near the melt/gas interface due to the Marangoni effect, incorporated with the free convection, enhances the counterclockwise vortex, and thus suppresses the clockwise flow 


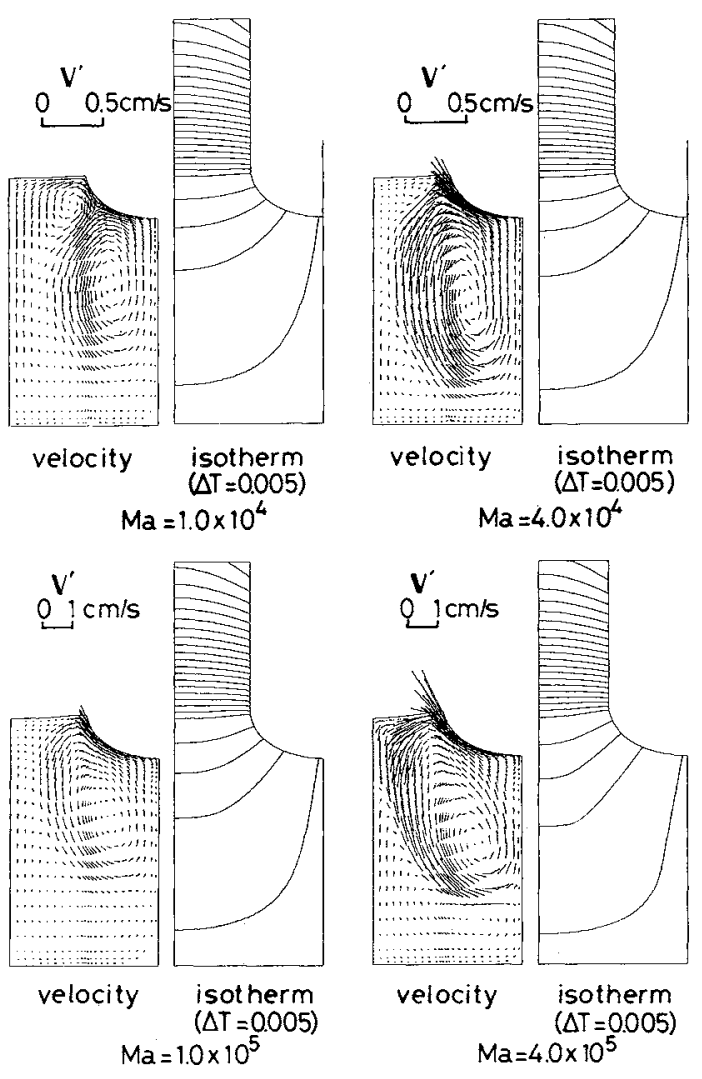

(a): Silicon with $G r=1.8 \times 10^{6}, R e_{5}=500, R e_{c}=0$ and $T_{c}=$ 1.0207

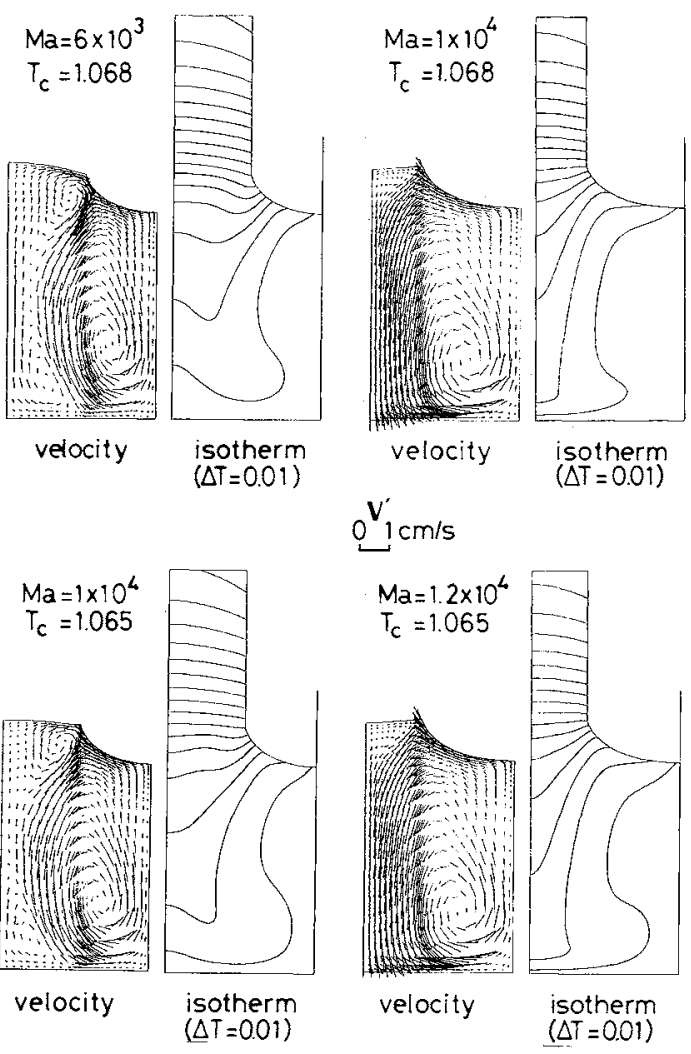

(b): Sapphire with $G r=8.56 \times 10^{5}, R e_{s}=700$ and $R e_{c}=0$

Fig. 7. Effect of Marangoni convection. caused by crystal rotation. With large $M a(>1.0 \times$ $10^{5}$ ) in silicon crystal growth, the counterclockwise flow mostly caused by Marangoni convection prevails in the upper part of the melt pool and leaves a slow circulating flow near the crucible bottom. In the case of $\mathrm{Al}_{2} \mathrm{O}_{3}$ with larger $\mathrm{Pr}$ and at higher temperature level, however, a large voltex reaching down to the bottom is made up by both the thermocapillary and the buoyancy effects in concert.

Figures 8(a) and (b) show the effect of $M a$ on the melt/crystal interface shape and the crystal radius, respectively, where $\Delta z$ is that at the center line, $r=0$. For $\mathrm{Si}$, the melt crystal interface becomes slightly more convex to the melt and the crystal radius becomes smaller as $M a$ increases. For $\mathrm{Al}_{2} \mathrm{O}_{3}, \Delta z$ is kept at an almost constant positive value (convex to crystal) at $0 \leq M a \leq 7 \times 10^{3}$. In this range of $M a$, the radius of the crystal rod is also insensitive to $\mathrm{Ma}$. But with further increase of $M a$, the radius shows a steep decrease and, at the same time, $\Delta z$ changes its sign, i.e. reverses from convex to concave to crystal. If the crucible temperature is kept constant, the crystal radius becomes smaller and may disappear at far larger Marangoni numbers. Then, to increase the crystal radius, the crucible temperature must be reduced ${ }^{22)}$ In Fig. 8, some results under reduced crucible temperatures are also shown.

With the present method of calculation and number of elements, we could not obtain converged solutions for values of $M a$ larger than those corresponding to the right-most plot points shown in Fig. 8. If the reported values of $\left(\partial \gamma / \partial T^{\prime}\right)^{14,15)}$ are adopted to estimate realistic values of $M a$ for a $20 \mathrm{~mm}$-dia. crucible, $\mathrm{Ma}$ is expected to be as large as $2.7 \times 10^{7}$ for silicon and $5.7 \times 10^{5}$ for $\mathrm{Al}_{2} \mathrm{O}_{3}$. Accordingly, in the real melt pool, the effect of Marangoni convection is expected to be more remarkable than that shown in Fig. 7. Present calculations reveal that the Marangoni effect plays the most important role among the driving mechanisms to make up the flow field in a small pool of melt, even in terrestrial gravity. But, it should be noticed, as reported by many authors, ${ }^{1,5)}$ that the Marangoni effect is easily inhibited by the presence of a small amount of contaminants. Although there is no report on contaminants for melts of silicon or $\mathrm{Al}_{2} \mathrm{O}_{3}$, further experimental investigations are required.

In our previous studies, much attention was paid to the shape of the melt/crystal interface. However, the flow pattern itself also has importance. The incorporation of dopants and/or impurities from the melt is strongly affected by the flow field very close to the melt/crystal interface and also by the extent of mixing in the melt. The method of flow analysis proposed here will provide a more realistic flow field in the melt than do the previous methods and will help provide a 


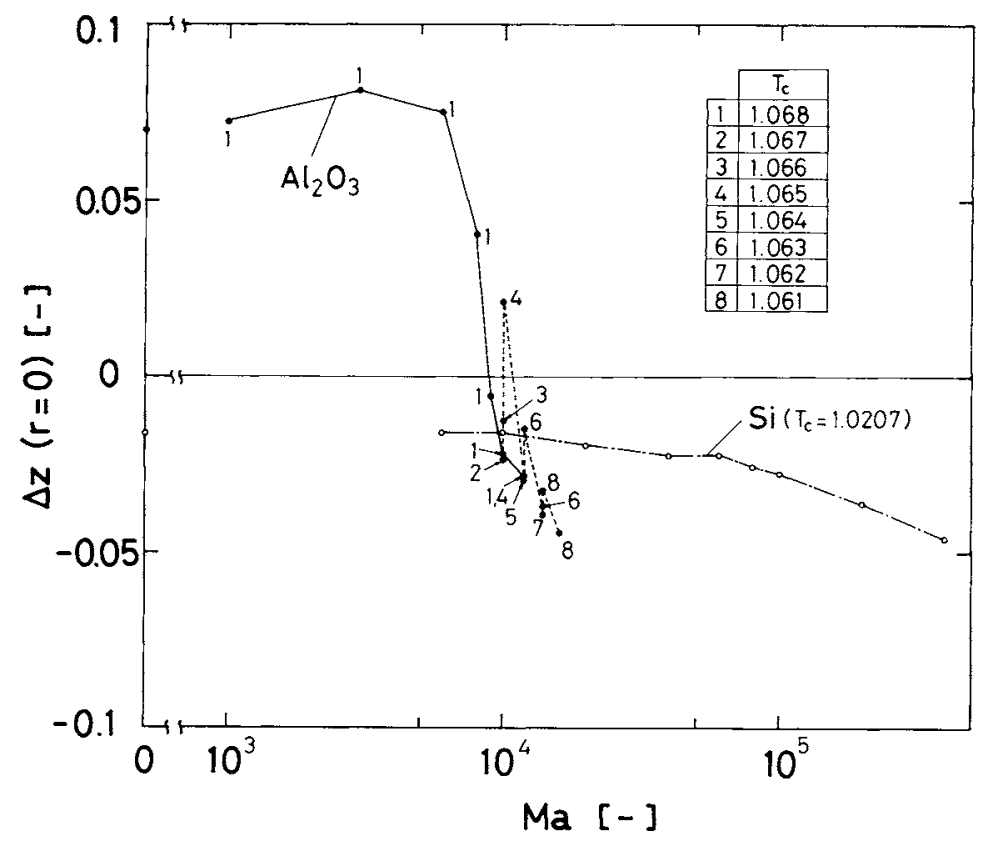

(a): Melt/crystal interface shape

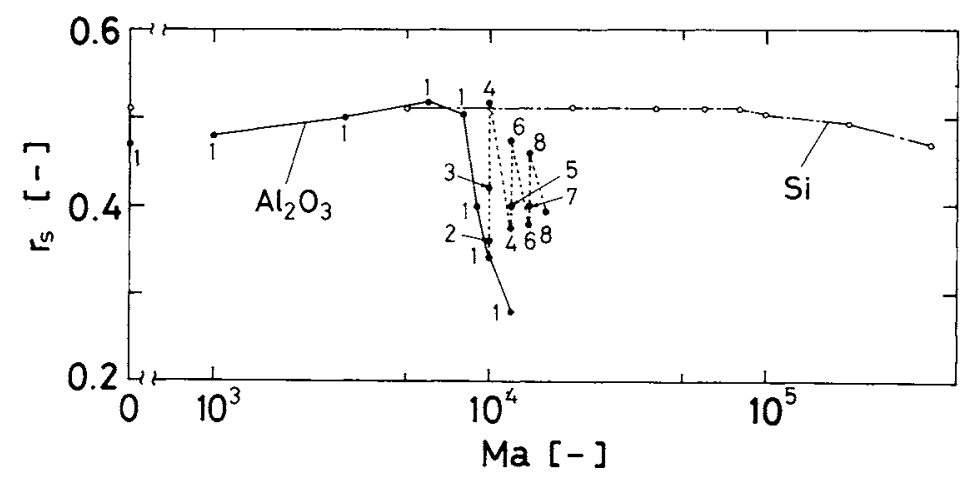

(b): Crystal radius

Fig. 8. Effect of Marangoni convection on melt/crystal interface shape and crystal radius. For silicon: $R e_{s}=500 ; R e_{c}=0 ; T_{c}=1.0207 ; G r=1.8 \times 10^{6}$. For sapphire: $R e_{s}=700 ; R e_{c}=0 ; G r=8.56 \times 10^{5}$.

deeper understanding of $\mathrm{CZ}$ crystal growth operations. To predict the flow of melt in large-scale industrial $\mathrm{CZ}$ equipment, however, the numerical scheme in solving the Navier-Stokes equation employed in the present analysis is not directly applicable, because of its fundamental assumptions of steady state and axial symmetry.

\section{Conclusions}

For silicon and $\mathrm{Al}_{2} \mathrm{O}_{3}$ crystal growth systems, the flow and temperature fields in the melt and the crystal having non-flat melt/crystal and melt/gas interfaces were studied theoretically and the following conclusions were obtained.

The theoretical method described here can predict the flow pattern in the melt, the temperature distributions in the melt and the crystal, the crystal radius and the shapes of both melt/crystal and melt/gas interfaces, although calculation was restricted here to the case of a small pool of melt.

For a fluid of small Prandtl number, such as silicon melt, the temperature distribution and the melt/ crystal interface are not sensitively affected by the flow field, however, those for $\mathrm{Al}_{2} \mathrm{O}_{3}$ with relatively large $\mathrm{Pr}$ are influenced by the flow field.

The Marangoni effect is, if it operates, the most important factor in promoting convection, at least in a small pool of melt such as is treated in the present analysis.

\section{Acknowledgments}

This work was supported by a Grant-in-Aid for Scientific Research 61550696 from the Ministry of Education, Science and Calture of Japan. The numerical calculations were carried out by use of ACOS 1000 and SX-1 of the Computer Center of Tohoku Univ.

\section{Nomenclature}

$A_{k}^{\prime} \quad=$ area of surface element $\left[\mathrm{m}^{2}\right]$ 


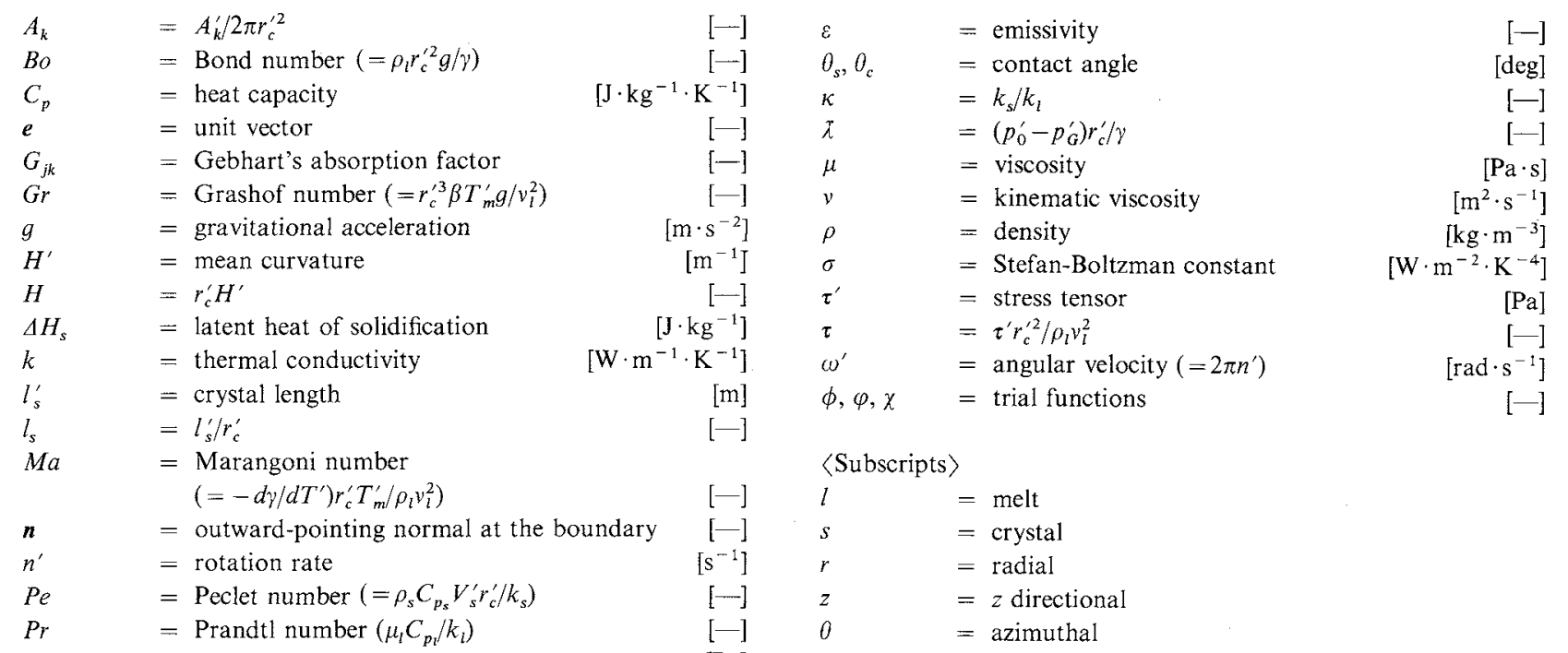

$p^{\prime} \quad=$ pressure $\quad[\mathrm{Pa}]$

$p_{G}^{\prime}=$ pressure in gas phase $\quad[\mathrm{Pa}]$

$\begin{array}{lll}p_{0}^{\prime} & =\text { pressure at reference position } & {[\mathrm{Pa}]}\end{array}$

$p \quad=\left(p^{\prime}-p_{0}^{\prime}+\rho_{l} g z^{\prime}\right) r_{c}^{\prime 2} / \rho_{l} v_{l}^{2} \quad[-]$

$q_{\text {rad }}^{\prime} \quad=$ heat flux due to radiation $\quad\left[\mathrm{W} \cdot \mathrm{m}^{-2}\right]$

$q_{\mathrm{rad}} \quad=q_{\mathrm{rad}}^{\prime} r_{c}^{\prime} / k^{\prime} \cdot T_{m}^{\prime} \quad[-]$

$R_{k} \quad=$ radiation number $\left(=\varepsilon_{k} \sigma r_{c}^{\prime} T_{m}^{\prime 3} / k_{k}\right) \quad[-]$

Re $\quad=$ Reynolds number $\left(=r_{c}^{\prime 2} \omega^{\prime} / v_{l}\right)$ [-]

$r^{\prime} \quad=$ radial distance in cylindrical coordinates $\quad[\mathrm{m}]$

$r_{c}^{\prime} \quad=$ crucible radius $\quad[\mathrm{m}]$

$r_{s}^{\prime} \quad=$ crystal radius $\quad[\mathrm{m}]$

$r, r_{s} \quad=r^{\prime} / r_{c}^{\prime}, r_{s}^{\prime} / r_{c}^{\prime} \quad$ [-]

St $\quad=$ Stefan number $\left(=\Delta H_{\mathrm{s}} / C_{p_{s}} T_{m}^{\prime}\right)$

$T^{\prime} \quad=$ temperature $\quad[\mathrm{K}]$

$T_{c}^{\prime} \quad=$ crucible temperature $\quad[\mathrm{K}]$

$T_{m}^{\prime} \quad=$ melting-point temperature $\quad[\mathrm{K}]$

$T_{a}^{\prime} \quad=$ enclosing-wall temperature $\quad[\mathrm{K}]$

$T, T_{c}, T_{a}=T^{\prime} / T_{m}^{\prime}, T_{c}^{\prime} / T_{m}^{\prime}, T_{a}^{\prime} / T_{m}^{\prime}$

$\Delta T \quad=$ interval of isotherms $\quad[--]$

$\boldsymbol{t}=$ tangential unit vector $\quad[-]$

$u^{\prime} \quad=$ radial component of velocity vectors $\quad\left[\mathrm{m} \cdot \mathrm{s}^{-1}\right]$

$V_{m}^{\prime} \quad=$ melt volume $\quad\left[\mathrm{m}^{3}\right]$

$V_{m} \quad=V_{m}^{\prime} / 2 \pi r_{c}^{\prime 3} \quad[-]$

$V_{s}^{\prime} \quad=$ crystal pull rate $\quad\left[\mathrm{m} \cdot \mathrm{s}^{-1}\right]$

$v^{\prime} \quad=$ velocity vectors $\quad\left[\mathrm{m} \cdot \mathrm{s}^{-1}\right]$

$v^{\prime} \quad=$ axial component of velocity vectors $\quad\left[\mathrm{m} \cdot \mathrm{s}^{-1}\right]$

$w^{\prime} \quad=\underset{\text { vectors }}{\text { azimuthal component of velocity } \quad\left[\mathrm{m} \cdot \mathrm{s}^{-1}\right]}$

$\begin{aligned} u, \boldsymbol{v}, v, w= & u^{\prime} r_{c}^{\prime} / v_{l}, \boldsymbol{v}^{\prime} r_{c}^{\prime} / v_{l}, v^{\prime} r_{c}^{\prime} / v_{l}, \\ & w^{\prime} r_{c}^{\prime} / v_{l}\end{aligned}$

$z^{\prime}=$ axial distance in cylindrical coordinates $\quad[\mathrm{m}]$

$z_{c}^{\prime} \quad=$ crucible height

$z, z_{c} \quad=z^{\prime} / r_{c}^{\prime}, z_{c}^{\prime} / r_{c}^{\prime}$

$z_{1}^{\prime}=$ distance between crucible bottom and melt/crystal interface

$z_{2}^{\prime}=$ distance between crucible bottom and melt/gas interface

$z_{1}, z_{2}=z_{1}^{\prime} / r_{c}^{\prime}, z_{2}^{\prime} / r_{c}^{\prime}$

$\Delta z^{\prime} \quad=z_{1}^{\prime}-z_{2}^{\prime}\left(r^{\prime}=r_{s}^{\prime}\right)$

$\Delta z \quad=\Delta z^{\prime} / r_{c}^{\prime}$

$\beta=$ thermal expansion coefficient

$\gamma \quad=$ surface tension

\section{Literature Cited}

1) Anderson, D.: IIW SG 212, Doc. 212-277-73, (1973).

2) Carruthers, J. R.: J. Crystal Growth, 36, 212 (1976).

3) Crochet, M. J., P. J. Wouters, F. T. Geyling and A. S. Jordan: J. Crystal Growth, 65, 153 (1983).

4) Derby, J. J., R. A. Brown, F. T. Geyling, A. S. Jordan and G. A. Nikolakopoulou: J. Electrochem. Soc., 132, 470 (1985).

5) Imaishi, N. and K. Fujinawa: J. Chem. Eng. Japan, 7, 87 (1974).

6) Kalejs, J. P., H. M. Ettouney and R. A. Brown: J. Crystal Growth, 65, 316 (1983).

7) Kobayashi, N. and T. Arizumi: Jpn. J. Appl. Phys., 9, 361 (1970).

8) Kobayashi, N. and T. Arizumi: Jpn. J. Appl. Phys., 9, 1255 (1970).

9) Kobayashi, N. and T. Arizumi: J. Crystal Growth, 30, 177 (1975).

10) Kobayashi, N.: J. Crystal Growth, 43, 357 (1978).

11) Kobayashi, N. and T. Arizumi: J. Crystal Growth, 49, 419 (1980).

12) Kobayashi, N.: J. Crystal Growth, 52, 425 (1981).

13) Langlois, W. E.: J. Crystal Growth, 42, 386 (1977).

14) Langlois, W. E.: J. Crystal Growth, 48, 25 (1980).

15) Langlois, W. E.: J. Crystal Growth, 56, 15 (1982).

16) Langlois, W. E.: J. Crystal Growth, 63, 67 (1983).

17) Mihelcic, M., C. Schroeck-Pauli, K. Wingerath, H. Wenzl, W. Uelhoff and A. van der Hart: J. Crystal Growth, 53, 337 (1981).

18) Mihelcic, M., C. Schroeck-Pauli, K. Wingerath, H. Wenzl, W. Uelhoff and A. van der Hart: J. Crystal Growth, 57, 300 (1982).

19) Mihelcic, M., K. Wingerath and Chr. Pirrou: J. Crystal Growth, 69, 473 (1984).

$[\mathrm{m}]$

$[-]$

$[-]$

[m]

$[-]$

$\left[\mathrm{K}^{-1}\right]$ $\left[\mathrm{N} \cdot \mathrm{m}^{-1}\right]$

20) Siegal, R. and J. R. Howell: Thermal Radiation Heat Transfer, McGraw-Hill, New York, 1982.

21) Srivastava, R. K., P. A. Ramachandran and M. P. Dudukovic: J. Crystal Growth, 73, 487 (1985).

22) Tsukada, T., N. Imaishi, M. Hozawa and K. Fujinawa: $J$. Chem. Eng. Japan, 20, 146 (1987).

23) Tsukada, T., N. Imaishi and M. Hozawa: J.Chem. Eng.Japan, in press. 